\title{
Aportaciones del Instituto Nacional de Investigaciones Forestales, Agrícolas y Pecuarias al mejoramiento genético de hortalizas
}

\author{
Enrique González-Pérez ${ }^{1 \S}$ \\ Moisés Ramírez-Meraz ${ }^{2}$ \\ Jaime Canul- $\mathrm{Ku}^{3}$ \\ Román Flores-López ${ }^{4}$ \\ Luis Martin Macías-Valdez ${ }^{5}$ \\ ${ }^{1}$ Campo Experimental Bajío-INIFAP. Carretera Celaya-San Miguel de Allende km 6.5, Colonia Roque, \\ Celaya, Guanajuato, México. CP. 38110. ${ }^{2}$ Campo Experimental Las Huastecas-INIFAP. Carretera \\ Tampico-Mante km 55, Cuauhtémoc, Tamaulipas, México. CP. 89610. (ramirez.moises@inifap.gob.mx). \\ ${ }^{3}$ Campo Experimental Zacatepec-INIFAP. Carretera Zacatepec-Galeana km 0.5, Zacatepec, Morelos, \\ México. CP. 62780. (canul.jaime@inifap.gob.mx). ${ }^{4}$ Sitio Experimental Metepec-INIFAP. Carretera \\ Toluca-Zitacuaro km 4.5, Zinacantepec, Estado de México, México. CP. 51350. \\ (flores.roman@inifap.gob.mx). ${ }^{5}$ Campo Experimental Pabellón-INIFAP. Carretera Aguascalientes- \\ Zacatecas km 32.5, Pabellón de Arteaga, Aguascalientes, México. CP. 20660. (macias.luis@inifap.gob.mx). \\ ${ }^{\S}$ Autor para correspondencia: gonzalez.enrique@inifap.gob.mx.
}

\section{Resumen}

México es centro de origen y de diversificación de especies hortícolas. A través de la diversidad genética que se posee, se han desarrollado diversos materiales mejorados, en los que, el Instituto Nacional de Investigaciones Forestales, Agrícolas y Pecuarias ha sido un pilar muy importante, por ser esta, una de sus principales actividades. En el programa de mejoramiento genético de hortalizas se aplican diferentes estrategias genotécnicas relevantes para el incremento del rendimiento de bulbo, fruto y tubérculo, como la selección de clones en ajo, selección individual o hibridación en cebolla, pedigrí o de formación de líneas puras por autofecundación en chile; selección recurrente o hibridación en jitomate, selección clonal en papa, selección familial de medios hermanos maternos en tomate de cáscara, con las que se han generado líneas experimentales, clones, híbridos y variedades. Producto de esas investigaciones se han liberado 19 variedades de ajo, 10 de cebolla, 21 de chile, 29 de papa y 2 de tomate de cáscara en los 35 años del INIFAP. Variedades que han sido adoptadas por productores en las diferentes regiones agroecológicas de México. Sin embargo, a pesar de los esfuerzos que los fitomejoradores hacen por continuar con la generación de materiales mejorados, no se ha podido impactar en un mercado dominado por empresas trasnacionales, por lo que, es necesario que el INIFAP implemente estrategias de posicionamiento e invierta en la conservación y mejora genética del germoplasma que posee, lo que a futuro resultará en la obtención mejores materiales genéticos que contribuyan al beneficio de la salud humana.

Palabras clave: conservación, germoplasma, hortalizas, variabilidad genética, variedades.

Recibido: abril de 2021

Aceptado: junio de 2021 
En México, el mejoramiento genético en hortalizas se ha limitado a pocas especies, principalmente a cultivos de mayor interés culinario, con demanda constante en el mercado nacional e internacional y por la basta diversidad genética existente en especies como el ajo (Allium sativum L.), cebolla (Allium cepa L.), chile (Capsicum annum L.), jitomate (Solanum lycopersicum L.), papa (Solanum tuberosum L.) y tomate de cáscara (Physallis ixocarpa Brot.), cuya importancia económica radica en la exportación. En 2019, el rendimiento promedio ( $\mathrm{t} \mathrm{ha}^{-1}$ ) fue de 12.24 en ajo, 29.78 en cebolla, 34.74 en chile, 63 en jitomate, 30.12 en papa y 19.18 en tomate de cáscara (SIAP, 2020).

Rendimientos que están directamente relacionados con la estructura genética de las poblaciones. México, es centro de origen del chile y del tomate de cáscara y de diversidad genética de cultivos como el ajo, jitomate, cebolla y papa, por lo que dispone de germoplasma nativo o acriollado (Rivera, 2001; Pérez et al., 2005; Ramírez-Meraz et al., 2015; Tavitas y Hernández, 2017; González-Chavira et al., 2019) para generar materiales con alto potencial de rendimiento, con amplia adaptación a las diferentes regiones productoras, los recursos genéticos de estas cinco especies están ampliamente distribuidos en México.

En ajo, se conocen dos tipos Allium sativum L. var. Sativum (sofnecks; falso tallo blando o sin vástago floral) y Alliun sativum L. var. Ophioscordon (hardnecks; falso tallo duro o con vástago floral), de los cuales se ha generado la mayoría de los materiales que se cultivan en la región de El Bajío (Guanajuato y Querétaro), quienes producen ajo morado; la zona centro (Aguascalientes), con ajo blanco y la zona norte-centro (Zacatecas y Durango) con ajo jaspeado. Aunque también se produce en menor superficie en otros estados como Guerrero, donde los productores siembran genotipos criollos (Pérez et al., 2005).

En cebolla, en 1968 se inició la introducción intensiva de híbridos a México, con variedades de tipo blanca, granex, amarilla, granex amarilla, roja globo y roja, desde su introducción, la cebolla ha sufrido cambios en su constitución genética, lo que ha permitido la generación de variabilidad genética en materiales acriollados, que con el paso del tiempo han mostrado diferencias genéticas con sus antecesores por efecto del ambiente (Güemes y Inoue, 2001).

En México, se conocen cuatro tipos de cebolla: blanca, roja, amarilla y cebollines, donde las compañías trasnacionales tienen acaparado el mercado. Siendo así, los híbridos comerciales de cebolla más sembrados son importados como Cirrus, Carta Blanca y Cal 214 (comunicación personal con productores). En menor proporción se cultivan variedades de origen criollo, como Elena, Santa Teresa, La Chona, Santa Cruz, Copándaro, Cojumatlán y Estrella Roja (ATAG, 2015).

Para el caso de chile, del género Capsicum se conocen 30 especies, cinco de las cuales son domesticadas: $C$. annuum, $C$. frutescens, $C$. chinense, $C$. pubescens y C. baccatum. La especie más cultivada es $C$. annuum, seguida de $C$. frutescens (Wang y Bosland, 2006). México está considerado como el principal centro de origen y diversidad, y tiene la mayor variabilidad genética de la especie $C$. annuum (jalapeños, guajillos, serranos anchos, pasillas, de árbol, etc.); así también, se tienen otras especies de importancia como $C$. chinense (habanero), C. pubescens (chile perón o manzano) y $C$. frutescens del que solo se tienen materiales silvestres y semidomesticados (chilpayas o chiles tabasco) (Ramírez et al., 2015). 
En jitomate, México es centro de domesticación y diversificación (Peralta y Spooner, 2007). Posee amplia diversidad genética (Blanca et al., 2012) tanto de especies domesticadas como silvestres (Álvarez-Hernández et al., 2009; Chávez-Servía et al., 2011), además de materiales mutantes, recombinantes y exóticos. Las especies identificadas en México y con las que se ha trabajado mejoramiento genético son: $S$. lycopersicum, $S$. hirsutum, $S$. peruvianum, $S$. glandulosum y $S$. pimpinellifolium (Tavitas y Hernández, 2017; 2019). Siendo las especies emparentadas de $S$. lycopersicum, $S$. habrochaites y $S$. pimpinellifolium las únicas en las que se han realizado cruzas (Hernández-Bautista et al., 2014; Marín-Montes et al., 2020).

En papa, actualmente se cuenta con más de cuatro mil variedades a nivel mundial con una gran diversidad en cuanto a forma, color, sabor, resistencia a plagas y enfermedades y presentan diferentes niveles de adaptación a condiciones climáticas. Los recursos genéticos se conservan como colecciones de clones, tubérculos (in vivo), cultivos in vitro y en nitrógeno líquido, mientras que, la semilla botánica de especies silvestres se conserva en condiciones controladas, como en el Centro Internacional de la Papa (CIP) que cuenta con más de seis mil accesiones de variedades criollas, materiales silvestres y mejorados generados por el CIP y por otros programas nacionales.

En tomate de cáscara se han reportado más de 70 especies de Physalis de estas la más cultivada es Physalis ixocarpa Brot. (Physalis philadelphica Lam.), con ocho razas reconocidas y de la que se han derivado materiales comerciales (Peña et al., 2014). Dicho mejoramiento tiene como base la diversidad genética cuyas fuentes de germoplasma son silvestres, razas o cultivares primitivos, variedades antiguas, material que ha tenido un proceso de mejoramiento, pero que no ha sido liberado y germoplasma de especies introducidas que han evolucionado al nivel de acriollamiento.

Durante los 35 años de existencia del Instituto Nacional de Investigaciones, Forestales, Agrícolas y Pecuarias (INIFAP), investigadores del programa de mejoramiento genético de hortalizas de los diversos Campos Experimentales, han estado trabajado en el proceso de mejora genética de varias especies hortícolas implementado varios métodos genotécnicos, como la selección individual de bulbos y plantas, selección clonal de mutantes espontáneos o plantas para ajo (Lampasona, et al., 2003), selección individual, selección masal combinada, selección familial de medios hermanos e hibridación en cebolla (Güemes et al., 2010); selección individual, selección masal, masalestratificada, pedigrí, método de descendencia de una semilla, formación de líneas puras mediante autofecundación (SSD) para la formación de híbridos y el uso de haploidía en chile (Tut et al., 2013; Ramírez et al., 2018a; Santiago-López et al., 2018a; Santiago-López et al., 2018b; SantiagoLópez et al., 2020); selección recurrente, hibridación intra e interespecífica y selección genómica para jitomate (Marín-Montes et al., 2019; Hernández-Bautista et al., 2020), mejoramiento genético clásico, hibridación y selección clonal en papa (Rivera, 2001), selección masal visual estratificada, selección familial de medios hermanos maternos y cruzamientos planta a planta para tomate de cáscara (Peña et al., 2002; Santiaguillo et al., 2004), métodos de mejoramiento relevantes para el incremento del rendimiento de bulbo, fruto y tubérculo.

\section{Mejoramiento genético en ajo, cebolla, chile, jitomate, papa y tomate de cáscara en el INIFAP}

El área de mejoramiento genético de hortalizas de importancia económica en México es dominada por empresas trasnacionales como es el caso del jitomate, la cebolla, la papa y el tomate de cáscara, pero en especies como el ajo y el chile, México es competitivo. Lo que se refleja en la mayor superficie cultivada con variedades foráneas en los diferentes sistemas de producción del país. 
Ajo. En México, el mejoramiento genético de ajo inició en el ciclo otoño-invierno (OI) 1978-1979 en el Campo Agrícola Experimental Bajío, perteneciente al entonces Instituto Nacional de Investigaciones Agrícolas (INIA), actualmente INIFAP. Los trabajos se iniciaron con un proceso de selección clonal individual en la variedad de ajo chileno (utilizado para exportación) empleando como criterio principal la selección de bulbos con menor número de dientes con respecto al promedio de la variedad original, además del tamaño de bulbo, vigor y sanidad de la planta.

Después de seis ciclos consecutivos de selección se identificaron los seis mejores clones por selección individual y dos compuestos por selección masal. Clones que al ser evaluados respondieron favorablemente mostrando menor número de dientes por bulbo e incremento en el peso. En 1986, una selección masal fue liberada como variedad con el nombre de Chileno Compuesto 1 y en 1987 selecciones individuales con los nombres de Chileno Vikingo 1, Chileno Vikingo 2 y Chileno Apaseo con rendimientos de $16.5 \mathrm{t} \mathrm{ha}^{-1}$. El mismo criterio fue utilizado para el ajo tipo Taiwán (introducido en 1978), que después de seis ciclos se obtuvieron selecciones con 10 a 14 dientes por bulbo y rendimiento promedio de $41 \mathrm{t} \mathrm{ha}^{-1}$. Selecciones de las que se liberaron las variedades: Tacátzcuaro, Tingüindín, Tocumbo, Texcoco, Huerteño, Chapingo-94, INIFAP 94 y Celayense (Heredia y Heredia, 2000).

En Zacatecas, se liberó la variedad Barretero, obtenida a partir de germoplasma de la región de Chaparrosa, municipio de Villa de Cos, Zacatecas (Reveles-Hernández et al., 2014) y la variedad CEZAC 6, originada a partir de bulbos procedentes de Corea que mediante selección individual se obtuvo un genotipo del tipo jaspeado, provisionalmente identificada como coreano y como Jaspeado Calera Orión (Cuadro 1) (Reveles-Hernández et al., 2011). En Aguascalientes, se liberó la variedad Orión de ajo tipo perla, obtenida del clon CAL-RN-11-1-1-2-4 (material altamente productivo) (Macías et al., 2010 b) y por selección individual se obtuvo la variedad tipo perla San Marqueño proveniente del clon C-37-1/8 y Orión (Cuadro 1) (Macías et al., 2010 a).

Cuadro 1. Materiales mejorados de hortalizas liberados* por el INIFAP en la última década.

\begin{tabular}{cccc}
\hline Especie & Categoría & Tipo & Denominación \\
\hline Ajo & Variedad & Jaspeado & Barretero y CEZAC 6 \\
& Variedad & Perla & San Marqueño y Orión \\
& Variedad & California & Diamante \\
& Variedad & Blanca & Blanca Morelos \\
Cebolla & Variedad & Habanero & Jaguar, Mayapan y Calakmul \\
& Variedad & Ancho & AP-VR \\
& Variedad & Ancho & AM-VR \\
& mulato & Don Luis \\
& Hibrido & Serrano & HS-44. \\
& Híbrido & Ancho & HAP14F \\
& Híbrido & Ancho & HAM14F \\
& Híbrido & Guajillo & HAMG14E \\
\hline
\end{tabular}




\begin{tabular}{cccc}
\hline Especie & Categoría & Tipo & Denominación \\
\hline Papa & Variedad & Blanca & $\begin{array}{c}\text { Ramona, Nieder, Bayonera, Modesta, Milagros, } \\
\text { Granate, Cuarzo, Cristal, Plata, Nevada, Frisca, } \\
\text { Adelita, Paz, Antila, Real 14 y Micaela }\end{array}$ \\
& Variedad & Roja & Rubí y Citlali \\
Tomate de cascara & Variedad & Rosada & Ameyali \\
& Vorado & INI-181
\end{tabular}

*= Catálogo Nacional de Variedades Vegetales (CNVV).

Cebolla. La introducción intensiva de híbridos a México se inició en 1968 con los materiales White Álamo $\mathrm{F}_{1}$ y White Granex. En el mismo año, se iniciaron los primeros trabajos de mejoramiento para la formación de híbridos en lo que fue el INIA, ahora INIFAP. Trabajos que consistieron en la evaluación de materiales de cebolla de procedencia extranjera durante el ciclo O-I en el CAEB de Celaya, Guanajuato. Posteriormente, los trabajos de mejoramiento genético se enfocaron en la obtención de materiales resistentes a la floración prematura y al incremento del rendimiento, por ello, en 1976 se liberó el cultivar Santa Cruz recomendado para el ciclo primavera-verano (P-V) y en 1984 se liberó la variedad Géminis para el ciclo O-I en Guanajuato (Heredia, 1985).

En 1985 se inició el programa de mejoramiento en el CEBAJ de INIFAP, con el objetivo de generar variedades que reunieran características de tamaño, forma y rendimiento para exportación en siembras de O-I, lo que permitió en 1989 la liberación de las variedades Elena, Santa Teresa y Estrella Roja (Heredia, 1989). Por otro lado, en el Campo Experimental Zacatepec de 1996 a 2001 a partir de colectas de Copándaro y por selección familial de medios hermanos se liberó la variedad Blanca Morelos (Cuadro 1), que es la única que cuenta con título de obtentor vigente hasta 2027 (Güemes et al., 2010; Ayala et al., 2010).

Del año 2000 al 2012 en el INIFAP no se trabajó en mejoramiento genético de cebolla, lo que ocasionó la pérdida del germoplasma preservado en los programas de mejoramiento genético. Actualmente, en el CEBAJ se tiene una colección de 120 accesiones de cebolla blanca, morada y amarilla, en las que, desde 2012 se ha trabajado para la generación de variedades e híbridos de días intermedios, mediante cruzamientos de materiales con alto contenido de materia seca y compuestos funcionales, lo que permitió la obtención de 12 líneas avanzadas, que a partir de 2018 se han estado evaluando y de estas tres materiales promisorios en 2019 se evaluaron y caracterizaron en lotes comerciales, para su liberación como híbridos. A la fecha el INIFAP ha liberado 10 variedades.

Chile. En México, la investigación en chiles se inició en el Instituto de Investigaciones Agrícolas (IIA) y en la Oficina de Estudios Especiales (OEE) en los años 50's. Esta última, inició un programa de mejoramiento genético en 1952 que en 1956 se movió al Campo Experimental el Horno. Con la fusión del IIA y la OEE se formó el INIA en 1961, donde el proceso de mejoramiento genético se continuó en diferentes sedes: El CEBAJ para chiles anchos y mulatos; CE Pabellón para pasillas y guajillos; CE Las Huastecas, para serranos; CE Cotaxtla para jalapeños y el Campo Experimental Zona Henequenera para habaneros.

De ese esfuerzo inicial se generaron las primeras variedades de chile ancho (Esmeralda, Verdeño, Flor de Pabellón y Mulato V2), de pasilla (Pabellón y Apaseo), de guajillo (Loreto, La Blanca y Gran Mirasol), así como de chile serrano con la variedad Tampiqueño-74 que llegó a cubrir 90\% 
de la superficie nacional de chile serrano en los 70's (Pozo, 1981). En ese mismo periodo se conformó el banco de germoplasma de chile en el CEBAJ, con más de dos mil accesiones de los diferentes grupos raciales de Capsicum (Pozo, 1981).

Con esa variabilidad genética durante los 35 años de existencia de INIFAP, se han liberado 21 materiales de chile: dos variedades y tres híbridos de chile serrano, tres variedades y dos híbridos de habanero, tres variedades de jalapeño, dos variedades y dos híbridos de chile ancho, dos variedades y un híbrido de guajillo y una variedad de chile puya. De los cuales Coloso, Centauro, Don Benito, Jaguar, Mayapan, AP-VR, AM-VR y Don Luis (Cuadro 1) son los materiales genéticos con mayor impacto económico en México.

Jitomate. La mejora genética del jitomate en México no ha sido atendida por instituciones del gobierno y parcialmente por centros públicos de investigación. Solo las empresas trasnacionales lo han hecho de manera formal (Salgado-Meraz et al., 2018). El INIFAP tiene bancos de germoplasma de jitomate, del cual su personal investigador se ha limitado a regenerar y en el mejor caso caracterizar. El acervo genético de jitomate del INIFAP procede de diferentes lugares de México y del mundo, consta de 231 accesiones conformada por las especies S. lycopersicum, $S$. hirsutum, S. peruvianum, S. glandulosum y S. pimpinellifolium (Tavitas y Hernández, 2017, 2019), resguardadas en Zacatepec, Morelos y 129 accesiones conservadas en Celaya, Guanajuato (Molina y Córdova, 2006).

De estos, solo 54\% están caracterizados. En 1989, se inició un programa de mejoramiento genético en el Campo Experimental Zacatepec (CEZ) para generar variedades resistentes al chino del jitomate. Como resultado se obtuvieron cuatro líneas $\mathrm{F}_{7}$ tolerantes, sin liberar ninguna variedad (SNICS, 2020). De 2016 al 2019 se desarrollaron trabajos de mejora genética en colectas de jitomate realizadas a través de proyectos apoyados con recurso fiscal dando como resultado una colección de 25 accesiones, las cuales están siendo usadas para desarrollar materiales de tipo saladette, heirloom o rugoso y uva, de este último se tiene una línea avanzada que está en proceso de caracterización morfológica y fenotípica para su posible liberación como variedad. Sin embargo, con toda esa riqueza genética es mínima la formación de líneas avanzadas e híbridos experimentales (Hernández-Leal et al., 2013, 2019; Parra-Gómez et al., 2016) y en consecuencia a la fecha, el INIFAP no ha liberado materiales mejorados.

Papa. El programa de investigación de papa en México inició en 1949 en la OEE, auspiciado por la fundación Rockefeller, antecesora del INIA, quienes realizaron estudios sobre el tizón tardío y seleccionaron clones con resistencia a esta enfermedad (Niederhauser, 1954), lo que dio origen en 1958 a la variedad Eréndira. Posteriormente, se liberaron las variedades Anita, Berthita, Conchita, Dorita, Elenita, Florita, Gabriela, Greta, Hilda, Juanita, Murca, Atzimba y Montsama, altamente resistentes al tizón tardío, pero en algunos casos con poca calidad visual y de ciclo largo, características propias de las condiciones agroecológicas de las sierras de México.

Para la obtención de variedades se ha utilizado como base estudios epidemiológicos sobre el tizón tardío y la relación de este con especies silvestres nativas como $S$. Demisum, aptitud combinatoria general y específica de genotipos y variabilidad de la población (Zúñiga-López et al., 2000), la caracterización de razas del Oomiceto y los mecanismos de resistencia a este (Rubio et al., 2005). Así como, estudios sobre el complejo punta morada o zebra chip (Díaz-Valasis et al., 2014) y de la variabilidad genética de la papa con relación a la resistencia a haplotipos de Candidatus Liberibacter solanacearum (Hernández-Dehesa, 2018). 
Como resultado de esas investigaciones de los 58 materiales registrados en México en el Catálogo Nacional de Variedades Vegetales (CNVV) (SNICS, 2020), el INIFAP ha contribuido con 29 variedades, además de las 13 liberadas en la década de los 50's y 60's que no están en el CNVV. En 2014 se liberaron las variedades Nevada, Plata, Rubí, Granate, Cuarzo y Cristal. De 2015 al 2020 las variedades Citlali, Frica, Adelita, Paz, Antila, Micaela, Real 14y Ameyali (Cuadro 1). El programa de papa del INIFAP ha aportado genotipos con resistencia duradera a tizón tardío que han sido usados en programas de mejoramiento e incluso algunos genotipos se han liberado como variedades en otros países de Centroamérica.

Actualmente, se tienen dos bancos de germoplasma, uno in vivo con más de 5000 accesiones y otro in vitro que cuenta con 480 genotipos que tienen materiales con resistencia a tizón tardío, punta morada o zebra chip, nemátodo dorado, bacterias, precoces, con calidad industrial, tolerancia a altas temperaturas y amplia adaptabilidad (20 a $3400 \mathrm{~m}$ ) (López, 1988). De ambos bancos los mejoradores identifican de progenitores con cualidades deseadas en las nuevas progenies, seleccionan genotipos sobresalientes con el esquema de mejoramiento y evalúan en campo material libre de virus y otras enfermedades para que los genotipos seleccionados expresen todo su potencial de rendimiento.

Tomate de cáscara. Los trabajos de mejoramiento genético en tomate de cáscara iniciaron en 1972 en el CEZ del INIFAP, a partir de la evaluación de una colección de 49 poblaciones criollas del estado de Morelos.

En los cuatro años siguientes se evaluó las 11 mejores poblaciones y en el último año se identificó y seleccionó cuatro líneas de alto rendimiento, una de las cuales se liberó como 'Rendidora' (Saray et al., 1978) y a partir de la cual se han generado variedades inscritas en el CNVV que desafortunadamente han sido liberadas por otras instancias.

De 1980 al 2012 no se realizó mejoramiento genético en tomate de cáscara en INIFAP, lo que propició la pérdida de gran parte de la diversidad genética. Situación por la cual, durante este período los productores cultivaron materiales criollos que reproducían desde muchos años atrás (Magaña et al., 2011) y selecciones criollas de empresas extranjeras. A partir de 2014, se reactivó el programa de mejoramiento genético de esta especie, con la intención de aprovechar el reducido número de colecciones de germoplasma del INIFAP y de las colectas realizadas de 2014 al 2017.

Actualmente, el CEBAJ cuenta con 400 accesiones colectadas en regiones productoras de Guerrero, Estado de México, Morelos, Puebla y Tlaxcala, que a partir del 2014 se han utilizado para generar variabilidad genética. De estas accesiones se han derivado líneas experimentales con características deseables por los productores que están siendo evaluadas y de ests cinco están en etapas de evaluación de ciclos homólogos (González-Chavira et al., 2019). Una de ellas liberada en 2020 como variedad INI-181 (Cuadro 1) para su cultivo en la zona centro de México.

\section{Perspectivas y retos del mejoramiento genético de especies hortícolas en México}

Los cultivos hortícolas en México mantienen sus perspectivas de crecimiento a corto, mediano y largo plazo. Su principal fortaleza radica en el hecho de que, México cuenta con una gran diversidad genética en muchas de las especies hortícolas que mantienen una demanda constante por parte de la población y cuyos márgenes de utilidad son redituables. Su cultivo demanda 
inversiones considerables, siendo la semilla un insumo costoso y los precios obtenidos por el productor se ven afectados ante la concentración periódica que registra la oferta durante el año, por lo que, hacen uso de los recursos genéticos nativos o acriollados.

La alternativa es el uso de materiales mejorados por el INIFAP, adaptados a las diferentes regiones agroclimáticas de México y de menor costo en comparación a los materiales ofertados por las empresas trasnacionales, lo que traería beneficios directos a los productores y al INIFAP. La posibilidad de que productores de los estados donde se cultivan y comercializan hortalizas adopten los materiales genéticos mejorados del INIFAP, se puede lograr con la implementación de estrategias de promoción y difusión que proporcionen las condicionantes para que nuestras variedades eleven sus promedios de aceptación regional y por ende nacional. Reto que no es fácil de enfrentar, debido a que, en la actualidad alrededor de $95 \%$ de la semilla de especies hortícolas de importancia económica que se comercializan en México son de procedencia extranjera.

Es prioritario retomar los niveles de investigación para el desarrollo de nuevas variedades de ajo, cebolla, chile, jitomate, papa, tomate de cáscara y otras especies con demanda creciente para el consumo nacional e inclusive internacional. Además, de un mayor control de calidad en las semillas de variedades liberadas ya utilizadas por los productores, para evitar la pérdida de la biodiversidad del país y disminuir los costos de obtención de semillas de variedades de origen nacional.

El impulso y apoyo financiero del INIFAP al mejoramiento genético de hortalizas permitirá en el futuro cercano la obtención y liberación de materiales con características deseables por los productores y que sean competitivas. Es por esto, que la conservación y uso del germoplasma que el INIFAP posee es prioritario. El germoplasma resguardado en los diferentes campos experimentales, donde se realiza mejoramiento genético, es procedente de diferentes regiones agroecológicas ubicadas en estados como el Estado de México, Guanajuato, Oaxaca, Puebla, San Luis Potosí, Sinaloa, Tamaulipas, Tlaxcala y Yucatán. Colecciones núcleo con las que se sigue trabajando el mejoramiento genético tradicional que ha llevado a la obtención de líneas avanzadas derivadas del material élite y a la liberación de variedades.

Esfuerzo que ha sido insuficiente y limitado la competitividad del INIFAP ante las trasnacionales en el sector hortícola, por lo que, el reto es iniciar un programa sistematizado de mejoramiento genético aplicando diferentes estrategias genotécnicas. Para esto, se deberá innovar con materiales de selección genómica (Cappetta et al., 2020; Hernández-Bautista et al., 2020) combinadas con el mejoramiento tradicional, la hibridación, selección, retrocruzas, introgresión, etc.

Con el objetivo primordial de generar materiales mejorados adaptados a los diferentes sistemas de producción de México. Considerando factores restrictivos de tipo biótico y abiótico afectados por el cambio climático. A corto plazo, es importante continuar con el mantenimiento de los bancos de germoplasma, la generación y liberación de nuevas variedades hortícolas resistentes a plagas y enfermedades como un componente para la producción sustentable. Así como la producción de semilla de las variedades generadas en el INIFAP que satisfagan en forma oportuna la demanda cambiante de los usuarios.

En este sentido, se proyecta el registro de nuevos materiales como la variedad de chile serranillo o soledad CHISER-522 para la región del Golfo de México, el híbrido de chile serrano HS-52, la variedad de chile jalapeño rayado Crótalus desarrollada especialmente para la producción de chipotle, y la variedad de chile de árbol CdA- 58 para cubrir la demanda de productores del país 
(Ramírez et al., 2016; Ramírez et al., 2018b). Además, iniciará el programa de producción masiva de semilla de los híbridos HMG14E (chile guajillo), HAM14F (ancho mulato) y HAP14F (ancho poblano) de reciente generación (Santiago-López et al., 2018a; Santiago-López et al., 2018b; Santiago-López et al., 2020). En tomate de cascara prevé la liberación de la variedad INI-64, que es tomate morado recomendado para la zona centro de México.

En el mediano plazo, se deben incorporar tecnologías como la crio-preservación en bancos de germoplasma, continuar con el mejoramiento genético tradicional y asistido, la generación de variedades con resistencia a enfermedades, incorporar caracteres para tolerancia a diferentes temperaturas, calidad industrial, nutraceútica y para los diferentes usos culinarios y gourmet.

Continuar con la generación de tecnologías de producción sustentable y establecer programas de incremento de semilla de las variedades que satisfagan los requerimientos de toda la cadena productiva: el productor (rendimiento, resistencia a factores adversos, adaptación), el comercializador (calidad en apariencia, vida de anaquel), el procesador (rendimiento industrial) y el consumidor (formas, colores, aromas y sabores) (Ramírez et al., 2018b).

A largo plazo, el mantenimiento de los bancos de germoplasma y la generación de variedades deberá ser continuo y de alta prioridad, por lo que, es importante contar con el apoyo gubernamental e institucional para atender las demandas cambiantes de los productores y del sector productivo. Teniendo como premisa la conservación de los recursos genéticos y la generación de materiales que se adapten a los cambios del clima por efecto de las actividades del ser humano.

\section{Conclusiones}

El INIFAP tiene que revalorar la diversidad de germoplasma que posee. Debe enfocarse en la conservación del germoplasma y en la generación de materiales que demanda el productor y por ende el consumidor. Para lograr esto, es necesario refrescar el germoplasma sobresaliente existente en los bancos de germoplasma, continuar con colectas y plantear estrategias de frontera en la mejora genética en el futuro cercano.

\section{Agradecimientos}

Los autores agradecen ampliamente al Instituto Nacional de Investigaciones Forestales, Agrícolas y Pecuarias (INIFAP) por las facilidades prestadas para la realización de esta contribución.

\section{Literatura citada}

Álvarez-Hernández, J. C.; Cortez-Madrigal, H. y García-Ruiz, I. 2009. Exploración y caracterización de poblaciones silvestres de jitomate (Solanaceae) en tres regiones de Michoacán, México. Polibotánica. 28:139-159.

ATAG. 2015. Agenda técnica agrícola de Guanajuato. Cebolla de riego y temporal. Variedades. SAGARPA. Segunda edición. 344 p.

Ayala, A. S.; Güemes, G. M. J.; Aguilar, L. S. y Ayala, B. E. A. 2010. Adopción e impacto de la nueva variedad de cebolla blanca Morelos en el Estado de Morelos. Informe técnico. INIFAP-CIRPAS. Campo Experimental Zacatepec. Zacatepec, Méx. 35 p. 
Blanca, J.; Cañizares, J.; Cordero, L.; Pascual, L.; Diez, M. J. and Nuez, F. 2012. Variation revealed by SNP genotyping and morphology provides insight into the origin of the tomato. PLoS ONE. 7(10):48198. doi.org/10.1371/journal.pone.0048198.

Cappetta, E.; Andolfo, G.; Di Matteo, A.; Barone, A.; Frusciante, L. and Ercolano, E. 2020. Accelerating tomato breeding by exploiting genomic selection approaches. Plants. 9(9):1236. doi:10.3390/plants9091236.

Chávez-Servía, J. L.; Carrillo-Rodríguez, J. C.; Vera-Guzmán, A. M.; Rodríguez-Guzmán, E. y Lobato-Ortiz, R. 2011. Utilización actual y potencial del jitomate silvestre mexicano. SINAREFI-SAGARPA-CIIDIR-IPN e Instituto Tecnológico del Valle de Oaxaca. Oaxaca, Méx. 72 p.

Díaz-Valasis, M.; Cadena-Hinojosa, M. A.; Almeyda-León, I. H.; Carrillo Ramírez, J. R.; ArriagaCamarena, C. y Segura-León, O. 2014 a. Selección de genotipos de papa con tolerancia al pardeamiento asociado con la punta morada en campo y Bactericera cockerelli en invernadero. Rev. Cient. Agrop. Tuxpan. 2(2):105-110.

González-Chavira, M. M.; Guzmán-Maldonado, S. H.; Pons-Hernández, J. L.; Villalobos-Reyes, S. and González-Pérez, E. 2019. Genetic, chemical and agronomical characterization of husk tomato advanced lines. Agron. Mesoam. 30(1):101-114.

Güemes, G. M. J.; Ramírez, R. R.; Ayala, S. A. y Osuna, C. F. J. 2010. Cultivares de cebolla del INIFAP y comerciales en el estado de Morelos. Campo Experimental Zacatepec. SAGARPA-INIFAP. Morelos, México. Folleto técnico núm. 49. 23 p.

Güemes, G. M. y Inoue, K. 2001. Producción de semilla de alta calidad de tomate de cáscara variedad "Rendidora". Campo Experimental Zacatepec. INIFAP. Morelos, México. 7 p.

Heredia, Z. A. 1985. Géminis: nueva variedad de cebolla para el bajío. Campo Agrícola Experimental del Bajío. SARH-INIFAP. Celaya, Gto., México. Folleto técnico. 7 p.

Heredia, Z. A. 1989. Elena, Santa Teresa, Estrella Roja: nuevas variedades de cebolla para el bajío. Campo Experimental del Bajío. SARH-INIFAP. Celaya, Gto., México. Folleto técnico núm. 6.9 p.

Heredia, Z. A. y Heredia, G. E. 2000. El ajo en México, origen, mejoramiento genético y tecnología de producción. Campo Experimental Bajío, SAGAR, INIFAP. Celaya, Gto., México. Libro técnico núm. 3.102 p.

Hernández-Bautista, A.; Lobato-Ortiz, R.; Cruz-Izquierdo, S.; García-Zavala, J. J. y ChávezServía, J. L. 2014. Variación fenotípica, heterosis y heredabilidad de una cruza interespecífica de jitomate. Interciencia. 39(5):327-332.

Hernández-Bautista, A.; Lobato-Ortiz, R.; García-Zavala, J. J.; Cruz-Izquierdo, S.; Chávez-Servía, J. L.; Rocandio-Rodríguez, M.; Moreno-Ramírez, Y.; Hernández-Leal, E.; HernándezRodríguez, M. and Reyes-López, D. 2020. Prediction accuracy of genomic selection models for earliness in tomato. Chilean J. Agric. Res. 80(4):505-514. doi:10.4067/S071858392020000400505.

Hernández-Dehesa, G. M.; Rojas-Martínez, I. R.; Rivera-Peña, A.; Zavaleta-Mejía, E.; OchoaMartínez, L. D. and Carrillo-Salazar, A. 2018. Resistance in potato to two haplotypes of 'Candidatus Liberibacter solanacearum'. J. Plant Pathol. 100:191-196. doi.org/10.1094/Phyto-73-992.

Hernández-Leal, E.; Lobato-Ortiz, R.; García-Zavala, J. J.; Hernández-Bautista, A.; Reyes-López, D. and Bonilla-Barrientos, O. 2019. Stability and breeding potential of tomato hybrids. C. J. Agric. Res. 79(2):181-189. doi.org/10.4067/S0718-58392019000200181.

Hernández-Leal, E.; Lobato-Ortiz, R.; García-Zavala, J. J.; Reyes-López, D.; Méndez-López, A. y Bonilla-Barrientos, O. 2013. Comportamiento agronómico de poblaciones $\mathrm{F}_{2}$ de híbridos de tomate (Solanum lycopersicum L.). Rev. Fito. Méx. 36(3):209-215. 
Lampasona, S. G.; Martínez, L. and Burba, J. L. 2003. Genetic diversity among selected Argentinean garlic clones (Allium stivum L.) using AFLP (amplified fragment length polimorphism). Euphytica. 132(1):115-119. doi.org/10.1023/A:1024606004596.

López, D. H. 1988. Banco de germoplasma in vitro del programa de papa del Instituto Nacional de Investigaciones Forestales y Agropecuarias. Agric. Téc. Méx. 14:133-133.

Macías, V. L. M.; Maciel, P. L. H. y Silos, E. H. 2010a. "San Marqueño", una nueva variedad de ajo blanco. Investigación y Ciencia de la Universidad Autónoma de Aguascalientes. 18(49):4-11.

Macías, V. L. M.; Maciel, P. L. H. y Silos, E. H. 2010b. "Diamante": variedad de ajo tipo California para el Centro Norte de México. Campo Experimental Pabellón, INIFAP. Aguascalientes, Méx. Folleto técnico núm. 41.40 p.

Magaña, L. N.; Santiaguillo, H. J. y Grimaldo, J. O. 2011. Mejoramiento participativo de tomate de cáscara como estrategia de conservación in situ. Campo Experimental Valle de México. INIFAP. Estado de México, México. Folleto técnico núm. 48. 24 p.

Marín-Montes, I. M.; Lobato-Ortiz, R.; Carrillo-Castañeda, G.; Rodríguez-Pérez, J. E.; GarcíaZavala, J. J. y Velasco-García, Á. M. 2019. Riqueza alélica de poblaciones nativas de jitomate (Solanum lycopersicum L.) para el mejoramiento genético. Agrociencia. 53(3):355-370.

Marín-Montes, I. M.; Lobato-Ortiz, R.; Carrillo-Castañeda, G.; Rodríguez-Pérez, J. E.; GarcíaZavala, J. J.; Hernández-Rodríguez, M. and Velasco-García, A. M. 2020. Genetic parameters of an interspecific cross between $S$. lycopersicum L. and S. habrochaites Knapp \& Spooner. Rev. Chapingo Ser. Hort. 26(2):111-123. Doi: 10.5154/r.rchsh.2020.01.003.

Molina, M. J. C. y Córdova, T. L. 2006. Recursos fitogenéticos de México para la alimentación y la agricultura: informe nacional 2006. SAGARPA-SOMEFI. Chapingo, México. 172 p.

Niederhauser, J. S. 1954. Late blight in Mexico. Am. Potato J. 31:233-237.

Parra-Gómez, M. A.; Lobato-Ortiz., R.; García-Zavala, J. J.; Reyes-López, D. y Velasco-Alvarado, M. J. 2016. Evaluación de líneas de una cruza interespecífica de tomate. Rev. Fitotec. Méx. 39(1):59-65. doi:10.35196/rfm.2016.1.59-65.

Peña, L. A.; Molina, G. J.; Márquez, S. F.; Sahagún, C. J.; Ortiz, C. J. y Cervantes, S. T. 2002. Respuestas estimadas y observadas de tres métodos de selección en tomate de cáscara (Physalis ixocarpa Brot.). Rev. Fitotec. México. 25(2):171-178.

Peña, L. A.; Ponce, V. J.; Sánchez, C. F. y Magaña, L. N. 2014. Desempeño agronómico de variedades de tomate de cáscara en invernadero y campo abierto. Rev. Fitotec. Méx. 37(4):381-391. doi:10.35196/rfm.2014.4.381.

Peralta, I. E. and Spooner, D. M. 2007. History, origin and early cultivation of tomato (Solanaceae). In: genetic improvement of Solanaceous. Tomato. Razdan, M. K. and Mattoo, A. K. (Ed). Science Publishers. Enfield, New Hampshire, USA. 2(1):1-24.

Pérez, M. L.; Palemón, A. E.; Ayvar, S. S. y Cevallos, R. E. 2005. Adaptación de cultivares de ajo morado y blanco (Allium sativum L.) en Acatlán, Guerrero, México. Acta Universitaria. 15(1):55-65.

Pozo, C. O. 1981. Descripción de tipos y cultivares de chile (Capsicum spp.) en México. SARHINIA. Folleto técnico núm. 77. 40 p.

Ramírez, G. F.; Robledo, T. V.; Reyes, V. M.; Escobedo, B. L.; Torres, T. M. y García, O. H. 2015. Estudio histológico y morfológico de plantas autotetraploides y diploides de tomate de cáscara. Rev. Méx. Cienc. Agríc. 6(12):2291-2299. doi: 10.29312/remexca.v0i12.762. 
Ramírez, M. M.; Arcos C. G.; Méndez, A. R. y Meneses, M. I. 2018b. Variedades e híbridos de chile para el trópico de México. In: producción agropecuaria, un enfoque integrado. Meza, V. V. M. y Chay, C. A. J. (Ed.). Universidad del Papaloapan. México. 29-38 pp.

Ramírez-Meraz, M.; Arcos-Cavazos, G. y Méndez-Aguilar, R. 2018a. Jaguar, cultivar de chile habanero para México. Rev. Méx. Cien. Agr. 9(2):487-492. doi.org/10.29312/remexca. v9i2.1089.

Ramírez-Meraz, M.; Villalón-Mendoza, H.; Aguilar-Rincón, V. H.; Corona-Torres, T. y Latournerie-Moreno, L. 2015. Caracterización morfológica de chiles silvestres y semidomesticados de la Región Huasteca de México. Agroproductividad. 8(1):9-16.

Reveles-Hernández, M.; Velásquez-Valle, R. y Alvarado-Nava, M. D. 2011. CEZAC 06: variedad de ajo jaspeado para la región norte centro de México. Campo Experimental Zacatecas, CIRNOC-INIFAP. Calera, Zacatecas, México. Folleto técnico núm. 33.47 p.

Reveles-Hernández, M.; Velásquez-Valle, R. y Cid-Ríos, J. A. 2014. Barretero: variedad de ajo jaspeado para Zacatecas. Campo Experimental Zacatecas, CIRNOC-INIFAP. Calera, Zacatecas, Méx. Folleto técnico núm. 6. 132 p.

Rivera, P. A. 2001. Metodologías tradicionales usadas en el mejoramiento genético de papa en México. Campo Experimental Valle de México. SAGARPA-INIFAP. Toluca, Estado de México, México. Libro técnico núm. 3. 45 p.

Rubio-Covarrubias, O. A.; Douches, D. S.; Hammerschmidt, R.; da Rocha, A. and Kirk, W. W. 2005. Effect of temperature and photoperiod on symptoms associated with resistance to Phytophthora infestans after leaf penetration in susceptible and resistant potato cultivars. Amer. J. Pot. Res. 82(2):139-146.

Salgado-Meraz, L.; Lobato-Ortiz, R.; Pérez-Flores, L. J.; Cruz-Izquierdo, S.; Peña-Valdivia, C. y García-Zavala, J. J. 2018. Diversidad agronómica de poblaciones de jitomate tipo "Cherry" S. lycopersicum L. y S. pimpinellifolium L. con potencial en el mejoramiento genético. Rev. Fitotec. Méx. 41(4):499-507.

Santiago-López, U.; Ramírez-Meraz, M. y Méndez-Aguilar, R. 2018a. HAP14F, Híbrido de chile ancho poblano para el altiplano de México. Rev. Mex. Cienc. Agríc. 9(2):481.485. doi: 10.29312/remexca.v9i4.1405.

Santiago-López, U.; Ramírez-Meraz, M. y Méndez-Aguilar, R. 2018b. HAM14F: híbrido de chile ancho mulato para el altiplano norte-centro de México. Rev. Mex. Cienc. Agríc. 9(4):887892. doi: doi.org/10.29312/remexca.v9i4.1405.

Santiago-López, U.; Ramírez-Meraz, M. y Méndez-Aguilar, R. 2020. HMG14E, híbrido de chile guajillo para el altiplano de San Luis Potosí, México. Rev. Fitotec. Mex. 43(2):243-244. doi:doi.org/10.35196/rfm.2020.2.243.

Santiaguillo, H. J.; Cervantes, S. T. y Peña, L. A. 2004. Selección para rendimiento y calidad de fruto de cruzas planta x planta entre variedades de tomate de cáscara. Rev. Fitotec. Mex. 27(1):85-91.

Saray, M. C.; Palacios, A. A. y Villanueva, N. E. 1978. "Rendidora" Nueva variedad de tomate de cáscara. Campo Experimental Zacatepec. INIA-SARH. Zacatepec, Morelos, México. 8 p.

SIAP. 2020. Servicio de Información Agroalimentaria y Pesquera. Producción agrícola, cíclicos y perenes modalidad riego más temporal. https://nube.siap.gob.mx/cierreagricola/.

SNICS. 2020. Servicio Nacional de Inspección y Certificación de Semillas. Catálogo Nacional de Variedades Vegetales. https://datastudio.google.com/reporting/5b7206ba-e190-48fe9696-73523bfccf58/page/itBWB. 
Tavitas, F. L. y Hernández, A. L. 2017. Banco de germoplasma de jitomate (Lycopersicon sp. L) (Solanum lycopersicon L.) del Campo Experimental Zacatepec. Campo Experimental Zacatepec, INIFAP. Zacatepec, Morelos, Méx. Folleto técnico núm. 104. 30 p.

Tavitas, F. L. y Hernández, A. L. 2019. Acopio y conservación de los recursos fitogenéticos en el Campo Experimental Zacatepec. Campo Experimental Zacatepec, INIFAP. Zacatepec, Morelos, México. Folleto técnico núm. 111. 27 p.

Tut, P. F. J.; Santamaría, B. F.; Zavala, L. M. J. y Mier, T. J. C. B. 2013. Características de materiales mejorados de chile habanero para la Península de Yucatán. Campo Experimental Mocochá-INIFAP. Mococha, Yucatán, México. Folleto técnico núm. 13.33 p.

Wang, D. and Bosland, P. W. 2006. The genes of capsicum. Hort. Sci. 41(5):1169-1187.

Zúñiga-López, L. N.; Molina, G. J. D.; Cadena, H. M. A. y Rivera, P. A. 2000. Resistencia al tizón tardío de la papa (Phytophthora infestans) en cruzamientos de cultivares y clones de papa (Solanum tuberosum L.) Rev. Méx. Fitopatol. 18(1):1-9. 